Gynäk. Rdsch. 1973;13:I-IV

\title{
Contents, Vol. 13, 1973
}

\section{Inhaltsverzeichnis}

No. 1

Editorial 1

Kurzfassung von Zeitschriftenartikeln

Meyerowitz, S.; Satloff, A. und Romano, J. (Rochester, N.Y.): Induzierter

Abort aus psychiatrischer Indikation 3

Attal, B.; Gillet, J.-Y.; Zurlinden, B.; Henry, E. und Colette, C. (Besançon):

Die Chromatographie der Phospholipide im Fruchtwasser. Ein Index für den

Reifezustand der Lunge beim Fetus 17

Bhagwanani, S. G.; Fahmy, D. und Turnbull, A. C. (Cardiff): Vorhersage des

Atemnotsyndroms bei Neugeborenen durch Bestimmung des Lezithingehaltes im Fruchtwasser 22

Hobbins, J. C; Brock, W.; Speroff, L.; Anderson, G. G. und Caldwell, B.

(New Haven, Conn.): L/S-Quotient zur Vorhersage des Reifezustandes (in

utero) der fetalen Lunge

Protheroe, C. (Newcastle upon Tyne): Wochenbettpsychosen. Eine Langzeitstudie

von 1927 bis 1961

39

Denoix, P.; Lacour, J.; Wolff, J. P.; Weiler, J. und Michel, G. (Villejuif): Klas-

sifizierung der Kollumkarzinome nach dem TNM-System, angewandt auf den

Begriff und die Behandlung von T2 44

Übersichtsreferat

Seidl, St. (Hamburg): Die diagnostische Konisation der Cervix uteri

Varia 79

No. 2

Kurzfassungen von Zeitschriftenartikeln Ueland, K.; Novy, M. J. und Metcalfe, J. (Portland, Oreg.): Die Hämodynamik

herzkranker Frauen in der Schwangerschaft und bei Belastung $\quad 81$

Ruoss, C. F. und Bourne, G. L. (London): Toxoplasmose in der Schwangerschaft 99

Kommentar 104

Prevedourakis, C. N.; Strigou-Charalambis, E.; Michalas, S. und Alvanou-

Iakovakis, M. (Athen): Intrauterines Bakterienwachstum während der Wehen-

tätigkeit 104

Dellenbach, P. (Strasbourg): Wiederbelebung des Neugeborenen bei metaboli-

scher Azidose 109

Inhaltsverzeichnis

HI

Tessier, F.; Binard, C; Merger, R. und Daguet, G. (Paris): Infektion des Neu-geborenen während und nach der Geburt, ausgenommen parasitäre und virale

Infektionen 113 
Kommentar

133

Krawczuk, A. und Sipowicz, I. (Lodz): II. Symposium für Gynäkologie des Kindesalters, Lodz 29.-30. September $1972 \quad 134$

Originalarbeiten Schmid, J. und Schwyter, H. (Zurich): Durchblutungsmessung der Plazenta mit Technetium99m 140

Slunsky, R. (Klosterneuburg): Untersuchungen über die Hemmung der thromboplastischen Wirkung des Fruchtwassers mit Trasylol 147

Bogdan, C; Szalmay, G. und Hirsch, H. A. (Basel): Weitere Erfahrungen mit der kryochirurgischen Behandlung gutartiger Portioveränderungen 153

Ohlenroth, G.; Steffen, U. und Mönkemeier, D. (Lübeck): Proliferative Wirkung

des Oestriols am Endometrium corporis uteri: Vergleich mit 17/?-Oestradiol . 155

No. 3

Kurzfassungen von Zeitschriftenartikeln Bracken, M. B. und Swigar, Mary E. (New Haven, Conn.): Welche Ursachen

führen zum verspäteten Ansuchen auf Schwangerschaftsabbruch? 161

Schulman, H. und Randolph, Georgia (Bronx, N.Y.): Na-Gehalt des Frucht

wassers und wehenlose Latenzzeit beim Salzabort 172

Frigoletto, F. D. und Pokoly, T. B. (Boston, Mass.): Elektrolytveränderungen bei

Aborteinleitung mit hypertoner Salzlösung 177

Brown, F. D.; Davison, E. C. und Phillips, L. L. (New York, N.Y.): Blutgerin-

nungsveränderungen nach hypertoner Salzinfusion zur (späten) Aborteinleitung 184 Gustavii, Björn und Brunk, Ulf (Lund): Der Effekt von intraamnialer und extra-

amnialer Injektion hypertoner Salzlösung auf die Plazenta beim therapeutischen Abort. Histologische Studie 191

Csapo, A. I.; Sauvage, J. P. und Wiest, W. G. (St. Louis, Mo.): Wirksamkeit und

Annehmbarkeit von Prostaglandin F2 $<$ x intravenös als Abortivum 198

Embrey, M. P.; Hillier, K. und Mahendran, P. (Oxford): Aborteinleitung mittels

extraamnialer Applikation von Prostaglandin E7/8 und F2C1 201

Barr, Wallace und Naismith, W. C. M. K. (Glasgow): Prostaglandin peroral zur

Geburtseinleitung 207

Originalarbeiten

Hiersche, H.-D. (Kaiserslautern): Wann entsteht Leben? 212

Dubrauszky, V. und Linka, F. (Würzburg): Endometriosen mit maligner Ent-

artung 219

Stoll, W. und Bader, P. (Zurich): Der Säure-Basen-Haushalt und die Sauerstoff-

versorgung des Feten unter subpartualer Trispufferinfusion 231

IV

Inhaltsverzeichnis

No. 4

Kurzfassungen von Zeitschriftenartikeln

Committee of Medical Aspects of Automotive Safety, American Medical Associa

tion: Automobilsicherheitsgürtel für Schwangere 241

Speer, D. P. und Peltier, L. F. (Kansas City, Kans.): Beckenfrakturen und

Schwangerschaft 243

Croxatto, H. B.; Diaz, S; Fuentealba, B.; Croxatto, H. D.; Carrillo, D. und

Fabres, C. (Santiago): Studien über die Dauer des Eitransportes in der mensch- 
lichen Tube. I. Das Zeitintervall zwischen Ovulation und Auffinden der Eizelle ím Uterus der gesunden Frau 253

Ablow, R. C. und Orzalesi, M. M. (New Haven, Conn.): Röntgenographische

Darstellung lokalisiert die Erkrankung der hylinen Membranen 268

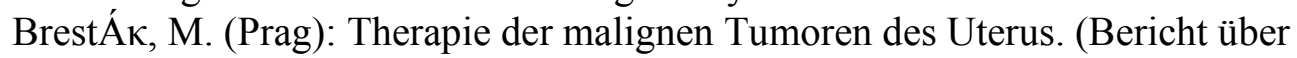
die Tagung der Tschechoslowakischen Gesellschaft für Gynäkologie, Brünn, 18. und 19. Mai 1972.) 275

Originalarbeiten Hilgarth, M.; Arbogast, R. und Hepp, H. (Freiburg/i. Br.): Tierexperimentelle Untersuchungen über den Einfluss von Clomiphen auf die Vaginalzytologie . . 293 Gigon, U.;

Keller, H. und Stamm, O. (St. Gallen): Einfluss eines synthetischen

Gonadotropin-Releasing-Hormons auf die Ausscheidung des luteinisierenden

Hormons (LH) und der Totalöstrogene bei sekundären Amenorrhoen .... 297 Nummi, S.;

Ylöstalo, P.; Korhonen, M. O. und Järvinen, P. A. (Oulu): Zytolo-

gische und histologische Befunde bei Intrauterinpessaren (Lippes-Loop) . . 307

Ergänzung zum Artikel von H. D. Hiersche : Wann entsteht Leben 314

Autorenindex 315

Supplementum

Schweizerische Gesellschaft für Gynäkologie

Bericht über die Jahresversammlung 28.-30. Juni 1973 in Basel

Société Suisse de Gynécologie

Compte rendu de ГAssemblée annuelle, 28.-30. Juni 1973 à Bale 